\title{
Two-photon photocurrent and voltage up-conversion in a quantum dot intermediate band solar cell
}

\author{
I. Ramiro, E. Antolín ，P.G. Linares, E. López, I. Artacho, A. Datas, A. Martí, and A. Luque
}

\begin{abstract}
It has been proposed that the use of selfassembled quantum dot (QD) arrays can break the ShockleyQueisser efficiency limit by extending the absorption of solar cells into the low-energy photon range while preserving their output voltage. This would be possible if the infrared photons are absorbed in the two sub-bandgap QD transitions simultaneously and the energy of two photons is added up to produce one single electron-hole pair, as described by the intermediate band model. Here, we present an InAs/Al $\mathbf{A}_{0.25} \mathrm{Ga}_{0.75} \mathrm{As} Q D$ solar cell that exhibits such electrical up-conversion of low-energy photons. When the device is monochromatically illuminated with $1.32 \mathrm{eV}$ photons, open-circuit voltages as high as $1.58 \mathrm{~V}$ are measured (for a total gap of $1.8 \mathrm{eV}$ ). Moreover, the photocurrent produced by illumination with photons exciting the valence band to intermediate band (VB-IB) and the intermediate band to conduction band (IB-CB) transitions can be both spectrally resolved. The first corresponds to the $Q D$ inter-band transition and is observable for photons of energy $>1 \mathrm{eV}$, and the later corresponds to the QD intra-band transition and peaks around $0.5 \mathrm{eV}$. The voltage up-conversion process reported here for the first time is the key to the use of the low-energy end of the solar spectrum to increase the conversion efficiency, and not only the photocurrent, of single-junction photovoltaic devices. In spite of the low absorption threshold measured in our devices - $0.25 \mathrm{eV}-$ we report open-circuit voltages at room temperature as high as $1.12 \mathrm{~V}$ under concentrated broadband illumination.
\end{abstract}

Index Terms - intermediate band solar cells, quantum dots, high-efficiency III-V solar cells, sub-bandgap photocurrent.

\section{INTRODUCTION}

The intermediate band solar cell (IBSC) [1] is a photovoltaic device designed to achieve a high efficiency through the exploitation of sub-bandgap photons. In this device, low energy photons are absorbed thanks to the existence of an extra band, the IB, within the semiconductor band gap of the absorber. As it is shown in Fig. 1 (a) this band enables two different transitions: VB-IB (labeled "1") and IBCB (labeled "2"). For the device to achieve high efficiency, it is of the utmost importance that both transitions are operative, and not just one of them (if we exclude impact ionization processes). In this respect, it is important that the IB is disconnected from the external contacts by the layers called "emitters". Since only electrons and holes exit the cell, and not IB carriers, it is not possible to extract photocurrent if only one sub-bandgap transition is used. In practice it may be possible to extract a current if the lattice thermal energy is used to sustain a carrier escape flux that replaces the failing optical transition (thermal escape). But in that case, the price paid is a reduction of the voltage. According to the IBSC theoretical model, by disconnecting the IB from the contacts and exploiting simultaneously the two sub-bandgap transitions, the IBSC can work at a voltage similar to the one it would have if no IB was present. That is the key of its high efficiency limit. Here we present our recent work to materialize those theoretical predictions in practice.

\section{EXPERIMENTAL}

We have fabricated and characterized IBSC prototypes with InAs/AlGaAs QD arrays as IB material [Fig. 1(b)]. They present improvements with respect to previous $\mathrm{QD}$ devices that make them exhibit a behavior closer to the ideal IBSC model. Their design includes the following features: (a) the total semiconductor band gap is enlarged through the use of $\mathrm{Al}$, blue-shifting $E_{\mathrm{G}}$ from $1.4 \mathrm{eV}$ (GaAs) to $1.7 \mathrm{eV}$ $\left(\mathrm{Al}_{0.25} \mathrm{Ga}_{0.75} \mathrm{As}\right)$; (b) the IB is shifted towards lower energies by introducing a strain-relief-layer on the QDs, red-shifting the inter-band QD transition (i.e. the VB-IB gap) from the typical value for InAs/(Al)GaAs QDs $\approx 1.1-1.2 \mathrm{eV}$ to $<1 \mathrm{eV}$.

These improvements to the design have been proposed in [2], where it was demonstrated that the thermal activation energy of these InAs/AlGaAs QDs is about $250 \mathrm{meV}$ higher than the energies found in the literature for InAs/GaAs QD, and almost $140 \mathrm{meV}$ higher than the activation energy obtained in our previous InAs/GaAs QD-IBSC prototypes including a strain-relief-layer. 


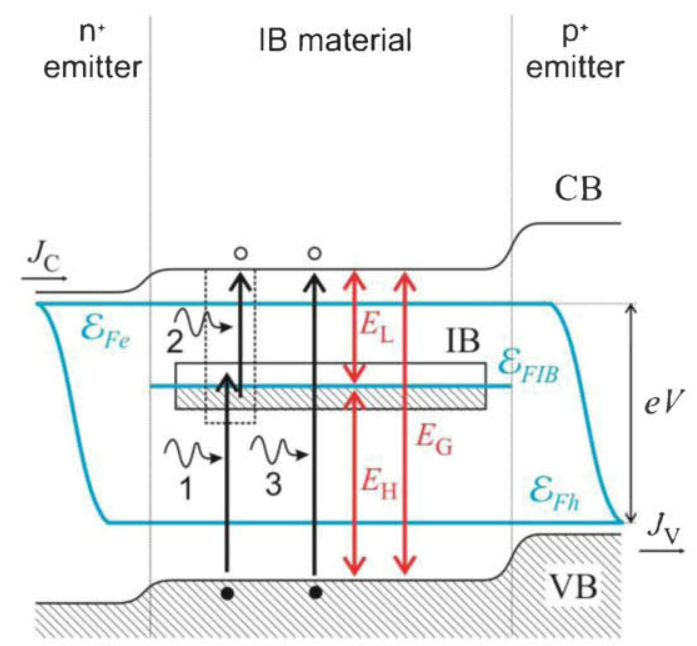

(a)

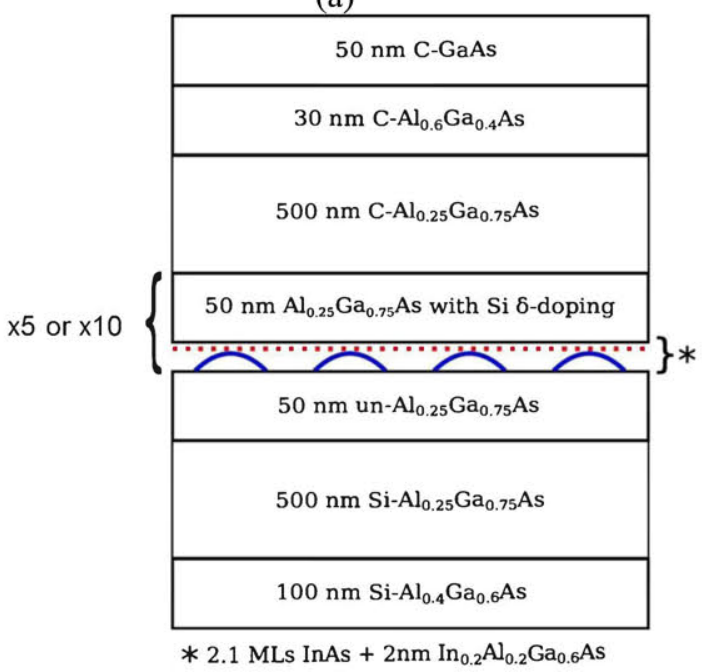

(b)

Fig. 1: (a) ideal band diagram of an IBSC under equilibrium. (b) layer structure of the InAs/AlGaAs IBSC prototypes studied in this work.

\section{RESULTS}

It has been possible to resolve spectrally the contribution of the IB-CB transition to the sub-bandgap photocurrent [Fig. 2(b)]. It produces a broad peak centered at $0.5 \mathrm{eV}$ (the dip is produced by atmospheric $\mathrm{CO}_{2}$ absorption). This result provides, for the first time, a complete characterization of the optical absorption properties of the QD-IBSC (comprising $E_{\mathrm{G}}$, $E_{\mathrm{H}}$ and $E_{\mathrm{L}}$ ) that had remained elusive in previous works. The measurement was performed at $40 \mathrm{~K}$ in the following way: when the IBSC is illuminated only with a monochromator, there is no photocurrent for photon energies lower than $1 \mathrm{eV}$ [red curve, Fig. 2 (b)] because these photons can produce IB$\mathrm{CB}$ transitions, but not VB-IB transitions. But when a laser of energy $1.32 \mathrm{eV}$ is illuminating the sample at the same time, its photons are absorbed in VB-IB transitions. Now carriers pumped by that laser to the IB can be transferred to the CB by the photons from the monochromator, which gives us the spectrally resolved photocurrent plotted in the blue curve, Fig. 2 (b).

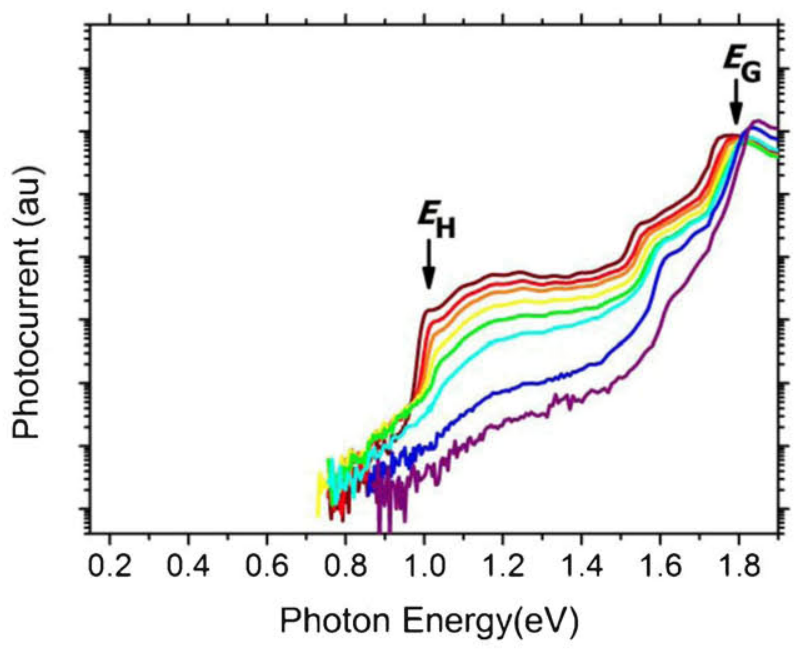

(a)

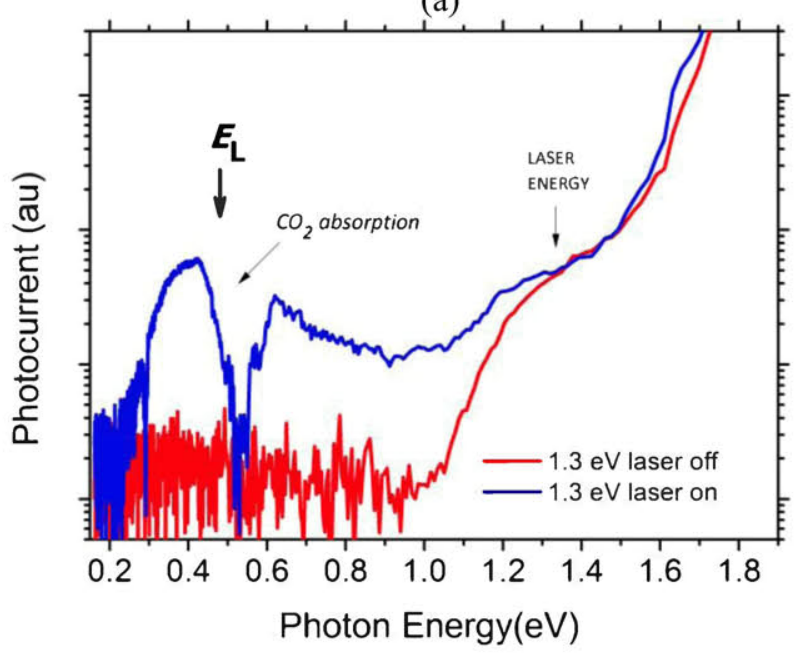

(b)

Fig. 2. Sub-bandgap photocurrent from a 10xQD-IBSC prototype. In (a) there is only one light source, a monochromator. The different curves represent different temperatures from $300 \mathrm{~K}$ (brown) to $100 \mathrm{~K}$ (purple). It can be seen how the photocurrent is suppressed as the IBCB thermal escape is reduced. (b) the red curve represents the same situation as above, now at $40 \mathrm{~K}$, and the blue curve is the photocurrent extracted when a dc laser of energy $1.32 \mathrm{eV}$ is shining on the cell. In this case the photons from the laser are used to pump electrons from the VB to the IB, while the light from the monochromator excites electrons from the IB to the $\mathrm{CB}$.

It has to be noted that the photocurrent measured shows a region of overlap of the absorption coefficients between 1.0 and $1.4 \mathrm{eV}$. Therefore, if we illuminate the device only with photons of energy $1.3 \mathrm{eV}$, they should be absorbed in both transitions. Fig. 3 shows the open-circuit voltage of the cells versus temperature for two different illuminations: in (a) the 
cell is illuminated with supra-bandgap photons and in (b) with $1.32 \mathrm{eV}$ photons. Both measurements provide a proof of voltage preservation ( $V_{\mathrm{OC}}$ is only limited by $E_{\mathrm{G}}$ and not by $E_{\mathrm{H}}$ or $E_{\mathrm{L}}$ ). But the result plotted in (b) has further significance because it implies that, due to the two-photon absorption process discussed above, this QD-IBSC prototype can produce a voltage higher than the energy of the photons used to illuminate it.

Finally, Fig. 4 shows an important achievement towards the implementation of a QD-IBSC which behaves as predicted by the IBSC model at room temperature. The $I V$ curves of two InAs/AlGaAs cells under are plotted, measured under concentrated broadband illumination and at room temperature. It can be seen that the open-circuit voltage is not limited by any of the sub-bandgaps at room temperature either.

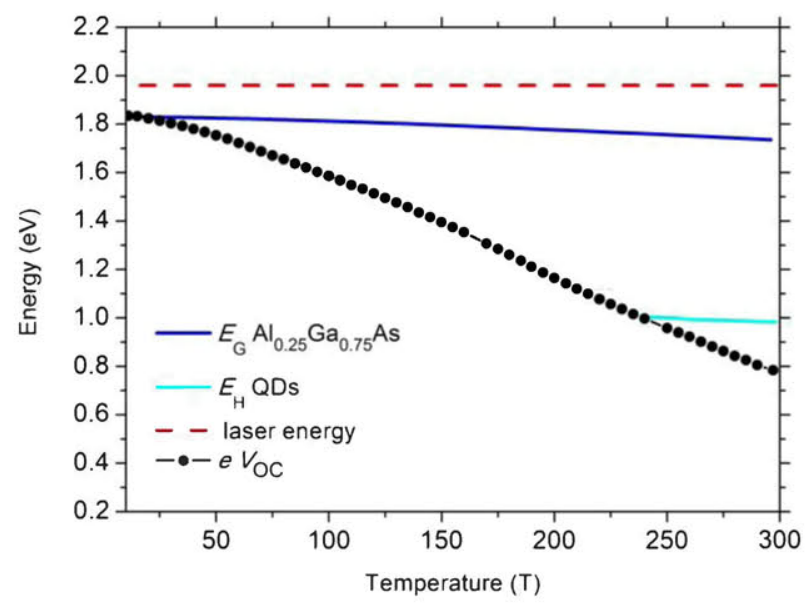

(a)

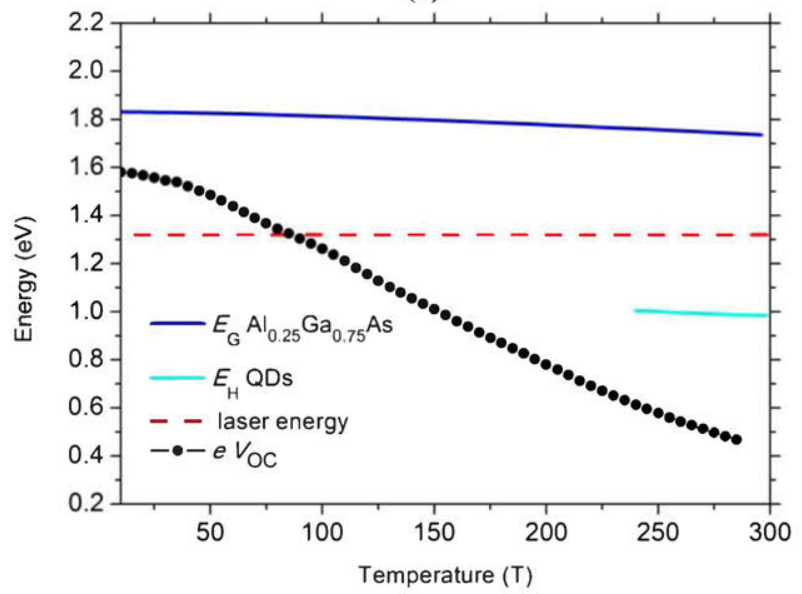

(b)

Fig. 3. Open-circuit voltage versus temperature, (a) for a 5xQD layer device illuminated with a laser of energy $1.96 \mathrm{eV}$, and (b) for a 10xQD device illuminated with a $1.32 \mathrm{eV}$ laser

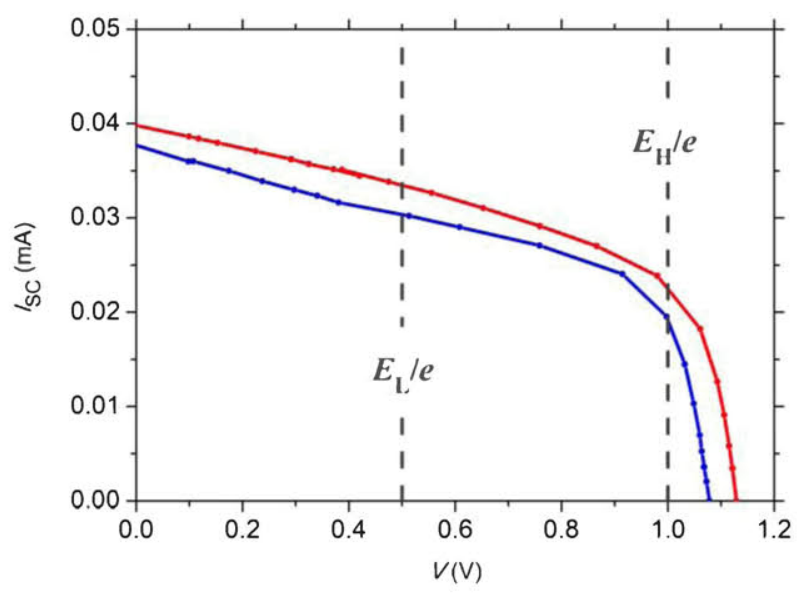

Fig. 4. Room temperature $I-V$ curves measured under concentrated broadband illumination, showing that the $V_{\mathrm{OC}}$ exceeds the two subbandgaps extracted from the photocurrent measurements (Fig. 2).The blue curve corresponds to a device with 10xQD layers measured at about 500 suns and the red curve to a 5XQD layer device measured at approximately 900 suns.

\section{SUMMARY}

In this work we have achieved crucial milestones in the development of a QD solar cell that realizes in practice the intermediate band solar cell model. We present a QD-IBSC that: (i) exhibits photocurrent produced by VB-IB and IB-CB transitions, fulfilling the requirement that the photocurrent can only be extracted when both transitions are excited (moreover, at low temperature we can obtain a spectrally resolved characterization of both transitions), (ii) exhibits voltage upconversion of low-energy photons, producing an open-circuit voltage of $1.6 \mathrm{~V}$ when it is illuminated with $1.3 \mathrm{eV}$ photons, and (iii) achieves at room temperature and under concentrated solar-like illumination an open-circuit voltages over $1.10 \mathrm{~V}$, which is $0.10 \mathrm{~V}$ and $0.80 \mathrm{~V}$ larger than the measured absorption thresholds (where photon energies have to be divided by $e$ to compare to voltage values).

\section{REFERENCES}

[1] A. Luque and A. Martí, "Increasing the efficiency of ideal solar cells by photon induced transitions at intermediate levels", Phys. Rev. Lett. 78, pp. 50145017, 1997.

[2] I. Ramiro, E. Antolin, M. J. Steer, P. G. Linares, E. Hernandez, et al., "InAs/AlGaAs quantum dot intermediate band solar cells with enlarged subbandgaps", in Photovoltaic Specialists Conference (PVSC), 2012 38th IEEE, pp. 652-656. 